\title{
BMJ Open Sexual counselling for patients with cardiovascular disease: protocol for a pilot study of the CHARMS sexual counselling intervention
}

\author{
Patrick J Murphy, ${ }^{1}$ Jenny Mc Sharry, ${ }^{1}$ Dympna Casey, ${ }^{2}$ Sally Doherty, ${ }^{3}$ \\ Paddy Gillespie, ${ }^{4}$ Tiny Jaarsma, ${ }^{5}$ Andrew W Murphy, ${ }^{6}$ John Newell, ${ }^{7}$ \\ Martin O'Donnell, ${ }^{7}$ Elaine E Steinke, ${ }^{8}$ Elaine Toomey, ${ }^{9}$ Molly Byrne ${ }^{1}$
}

To cite: Murphy PJ, Mc Sharry J, Casey D, et al. Sexual counselling for patients with cardiovascular disease: protocol for a pilot study of the CHARMS sexual counselling intervention. BMJ Open 2016;6:e011219. doi:10.1136/bmjopen-2016011219

- Prepublication history and additional material is available. To view please visit the journal (http://dx.doi.org/ 10.1136/bmjopen-2016011219).

Received 20 January 2016 Revised 4 April 2016 Accepted 21 April 2016

CrossMark

For numbered affiliations see end of article.

Correspondence to Patrick J Murphy; murphyp@nuigalway.ie

\begin{abstract}
Introduction: Sexual problems are common with cardiovascular disease, and can negatively impact quality of life. To address sexual problems, guidelines have identified the importance of sexual counselling during cardiac rehabilitation, yet this is rarely provided. The Cardiac Health and Relationship Management and Sexuality (CHARMS) intervention aims to improve the provision of sexual counselling in cardiac rehabilitation in Ireland.
\end{abstract}

Methods and analysis: This is a multicentre pilot study for the CHARMS intervention, a complex, multilevel intervention delivered within hospital-based cardiac rehabilitation programmes. The intervention includes (1) training in sexual counselling for staff, (2) a staff-led patient education and support intervention embedded within the cardiac rehabilitation programme, (3) a patient information booklet and (4) an awareness raising poster. The intervention will be delivered in two randomly selected cardiac rehabilitation centres. In each centre 30 patients will be recruited, and partners will also be invited to participate. Data will be collected from staff and patients/partners at T1 (study entry), T2 (3-month follow-up) and T3 (6-month follow-up). The primary outcome for patients/partners will be scores on the Sexual Self-Perception and Adjustment Questionnaire. Secondary outcomes for patients/ partners will include relationship satisfaction; satisfaction with and barriers to sexual counselling in services; sexual activity, functioning and knowledge; physical and psychological well-being. Secondary outcomes for staff will include sexuality-related practice; barriers to sexual counselling; self-ratings of capability, opportunity and motivation; sexual attitudes and beliefs; knowledge of cardiovascular disease and sex. Fidelity of intervention delivery will be assessed using trainer self-reports, researcher-coded audio recordings and exit interviews. Longitudinal feasibility data will be gathered from patients/partners and staff via questionnaires and interviews.

Ethics and dissemination: This study is approved by the Research Ethics Committee (REC) of the National University of Ireland, Galway. Findings will be disseminated to cardiac rehabilitation staff, patients/

\section{Strengths and limitations of this study}

- Our study focuses on an under-researched, sensitive and important area of healthcare provision: sexuality and the provision of sexual counselling.

- This pilot study protocol for our sexual counselling intervention in cardiac rehabilitation is based on significant exploratory research, recently published international guidelines for best practice in sexual counselling and the latest guidance on the development of complex interventions.

- The intervention is comprehensive (targeting cardiac rehabilitation staff as well as patients and their partners), and has been designed to be integrated sustainably into usual care.

- The protocol includes a thorough evaluation of feasibility and acceptability for the sexual counselling intervention using multiple complimentary methods.

- Self-selection bias into the pilot study may influence assessments of feasibility and acceptability for the intervention.

partners and relevant policymakers via appropriate publications and presentations.

\section{INTRODUCTION}

Cardiovascular diseases are the most common cause of morbidity and death globally. ${ }^{1}$ Sexual problems are more prevalent among individuals with cardiovascular disease than among the general population. $^{2}{ }^{3}$ Sexual problems negatively impact quality of life, psychological well-being and relationship satisfaction. ${ }^{4}{ }^{5}$ Sexual problems also impact patients' partners who rate sexual concerns as one of the most prevalent stressors. ${ }^{6}$

Reasons for the association between cardiovascular disease and sexual problems include physical vascular causes, ${ }^{7}$ fear of sexual 
activity provoking cardiac symptoms or a cardiac event, ${ }^{8}$ patient/partner relationship changes following a cardiac event $^{9}$ and associations with psychological problems such as depression. ${ }^{10}$ Sexual side effects of cardiac medications have been indicated, but recent research is less conclusive. ${ }^{11} 12$

A consensus document endorsed by the American Heart Association and the European Society of Cardiology recommends that all patients with cardiovascular disease be assessed for sexual concerns and offered sexual counselling as part of cardiac rehabilitation. ${ }^{12} 13$ Despite this endorsement and the fact that most people with cardiovascular disease are interested in receiving sexual education and counselling, ${ }^{14}$ sexual problems are rarely addressed during cardiac rehabilitation. ${ }^{15}$

How to best implement sexual counselling in cardiac rehabilitation is as yet uncertain. Very few trials in this area have been reported. There is some evidence that a highly intensive intervention (ie, 5 hours of sexual therapy provided by a sex therapist) can reduce the time taken to resume sexual activity following a cardiac event, and improve self-reported sexual function and satisfaction. ${ }^{16}$ However, it is unlikely that this type of intervention could be implemented as part of routine cardiac rehabilitation in most healthcare systems due to time and resource limits. Randomised controlled trials (RCTs) of time-efficient interventions are, therefore, needed. ${ }^{17}$

\section{The CHARMS baseline study}

The Cardiac Health and Relationship Management and Sexuality (CHARMS) baseline study conducted by the current research team examined the prevalence and treatment of sexual problems among patients $(\mathrm{N}=382)$ attending cardiac rehabilitation in Ireland. ${ }^{18}$ The CHARMS baseline study reported a high prevalence of sexual problems-sexual dysfunction was reported by $47 \%$ of sexually active male patients and $37 \%$ of sexually active female patients. Almost half $(47 \%)$ of all patients surveyed reported no sexual activity in the previous year, nearly double the rate for an age-matched non-cardiac sample. ${ }^{19}$ Less than one-third $(29 \%, n=114)$ of patients reported that sexual issues had been addressed in the cardiac rehabilitation programmes; among these patients, satisfaction with this aspect of the service was relatively low. Almost half of all patients $(48 \%, \mathrm{n}=183)$ would have liked more opportunity to discuss sexual issues with health professionals.

The CHARMS baseline study also surveyed nationally representative samples of general practitioners and cardiac rehabilitation staff. Health professionals seldom discussed sexual issues with patients, lacked awareness, knowledge and confidence in this area, and wanted more training and specific treatment guidelines. ${ }^{20} 21$

Finally, the CHARMS baseline study included focus groups with cardiac rehabilitation patients and staff. ${ }^{14}$ Analysis of these focus groups supported the results from the quantitative surveys, highlighting the disconnect between the identified need for support for sexual issues among patients, and the low level of service provision reported by staff. The possible components of a sexual counselling intervention were modelled. Staff were welcoming of additional training in sexual counselling guidelines, and patients were welcoming of the inclusion of support for sexual issues as a routine part of the cardiac rehabilitation programme.

\section{Development of the CHARIMS intervention}

Using the Behaviour Change Wheel (BCW) framework for the design and evaluation of behaviour change interventions,${ }^{22}$ the results from the CHARMS baseline study were used to develop the CHARMS intervention. This is a complex, multilevel intervention designed to increase the provision of sexual assessment and counselling in cardiac rehabilitation, and improve sexuality-related outcomes for patients with cardiac disease. It is delivered as an integrated element of hospital-based cardiac rehabilitation, and it addresses the main barriers to the provision of sexual counselling that were previously identified. The intervention includes (1) training and support for cardiac rehabilitation staff, (2) a staff-led patient intervention, (3) a patient information booklet and (4) an awareness raising poster for display in cardiac rehabilitation centres. The details of the development of the CHARMS intervention using the $\mathrm{BCW}$, and details of the specific behaviour change techniques (BCTs) associated with those components, are described elsewhere (McSharry J, Murphy PJ, Byrne M. Developing the CHARMS intervention to improve implementation of sexual assessment and counselling in hospital cardiac rehabilitation. Implementation Science, under review). An example of a summary mapping of BCTs and intervention components is provided in the online supplementary file. The finalised intervention components are described in detail below under the section 'Intervention procedures'.

\section{The CHARMS pilot study}

The most recent guidance from the Medical Research Council (MRC) in relation to complex interventions advises a 'development-evaluation-implementation' process. ${ }^{23}$ The development and piloting phases of trials are commonly skipped or minimised, with researchers proceeding directly to RCTs of newly developed interventions. ${ }^{24}$ This practice has been identified as contributing to the failure of many clinical trials to deliver conclusive results due to "[p]oor design choices, low levels of recruitment, significant attrition, lack of intervention fidelity, and interventions unacceptable to those delivering or receiving [them]". ${ }^{24}$ Pilot studies are essential in assessing the acceptability and feasibility of interventions and study protocols; these help to avoid the cost of failed trials. ${ }^{25}$ Accordingly, the aim of the CHARMS pilot study is to gather and analyse acceptability and feasibility data that will allow for (1) the refinement of the CHARMS intervention, and (2) the development of a definitive cluster RCT protocol to test this intervention. 
Specifically, the CHARMS pilot study will answer the following key research questions:

1. What is the likely effect size for the primary outcome among patients?

2. What is the optimal number of cardiac rehabilitation centres (clusters) and cardiac rehabilitation patients (participants) needed for a definitive cluster RCT?

A. What are the recruitment and attrition rates for clusters and participants?

B. What is an unbiased estimate of the intracluster correlation coefficient for the primary outcome among participants?

3. Is the intervention both feasible and acceptable?

A. Are the intervention components acceptable to staff and patients?

B. Are the outcome measures acceptable to staff and patients?

C. Will the intervention be delivered as designed (ie, can fidelity be assured)?

\section{METHODS AND ANALYSIS \\ Design}

The CHARMS pilot study is a multicentre feasibility study. In line with MRC guidance, the CHARMS pilot study will not be a 'scale model' of a definitive cluster RCT. However, the pilot will provide data that will 'address the main uncertainties that have been identified in the development work', ${ }^{23}$ and meet the aims and objectives outlined above. The CHARMS intervention will be implemented in two cardiac rehabilitation centres (clusters) in Ireland. At the cluster level, a 'CHARMS Educator' will deliver the CHARMS staff intervention, consisting of training and support for cardiac rehabilitation staff in the provision of sexual counselling to patients. At the participant level, trained cardiac rehabilitation staff will deliver education and support to patients and their partners. Following a repeated-measures design, quantitative and qualitative data will be gathered from staff, patients and partners at entry to the study T1 (baseline, preintervention assessment), at T2 (3 months after entry to the study, first postintervention assessment), and at T3 (6 months after entry to the study, second postintervention assessment).

\section{Participants}

Inclusion criteria for cardiac rehabilitation centres

There are 37 cardiac rehabilitation centres in the Republic of Ireland. ${ }^{26}$ Each of these centres operates as a unit within a hospital and most, but not all, offer phase III cardiac rehabilitation (ie, initial outpatient cardiac rehabilitation). While phase III cardiac rehabilitation programmes vary by centre, generally these start within 4-6 weeks of the patient's discharge from hospital and involve a rehabilitation programme of twice-weekly supervised exercise and group-based educational sessions on various topics related to living with cardiovascular disease. Programmes usually last for 8 weeks. Eligible centres for inclusion in the CHARMS pilot study will offer phase III cardiac rehabilitation, and will have a minimum of 80 patients attending rehabilitation per year (to ensure sufficient patient recruitment).

\section{Inclusion criteria for cardiac rehabilitation patients}

Eligible patients will be aged 18 years or older, will have cardiovascular disease and will be enrolled in phase III cardiac rehabilitation in the participating centres. Diagnosis of cardiovascular disease will not be confirmed from medical records as the patients cannot enrol into the cardiac rehabilitation programme without such a diagnosis. Eligible patients will be invited to participate in the study at enrolment to the cardiac rehabilitation programme. Sexual inactivity before entering cardiac rehabilitation will not be an exclusion criterion as previous behaviour is not an accurate predictor of future sexual behaviour or need for information, ${ }^{27}$ and even sexually inactive older adults and those who may be very ill value sexuality as an important part of life and health. ${ }^{28}$ Similarly, patients who do not have a current partner will not be excluded from participation as sexual self-satisfaction is an important part of sexual health for all people, regardless of relationship status. ${ }^{29}$

\section{Sample size calculations}

A function of the CHARMS pilot study is to gauge the feasibility of recruiting a sufficient number of clusters and participants for a definitive cluster RCT. Sample size calculations for this pilot are, therefore, based on calculations for the envisaged definitive trial, and a summary of the latter are presented here. The primary outcome for the definitive trial will be the Sexual Self-Perceptions and Adjustment Questionnaire (SSPAQ) which has been developed by Steinke $e t a l^{30}$ to measure psychosexual outcomes among cardiac patients. Given the absence of normative data for the Irish cardiac population to use as the base for an estimate of effect size for the SSPAQ, provisional sample size calculations for the definitive trial were based on normative data for a US cardiac sample. ${ }^{30}$ Based on this normative data, for the definitive trial, it is expected that a total of five clusters, each containing 22 participants randomly allocated per arm $(n=220)$, will be needed in order to have $80 \%$ power $(\alpha=0.05)$ to detect a difference in mean improvement of 10 units between the controls and those receiving the intervention, assuming a SD of 21 units in 6-month SSPAQ improvement in both arms and an intracluster correlation of 0.05 . Note that the trial is feasible as the total number of clusters, that is, 10 , is greater than $n_{1} \times r$ $(142 \times 0.05)=7$, where $n_{1}$ is the number of participants needed under individual randomisation and $r$ is the estimated intracluster correlation coefficient. ${ }^{31}$ To allow for estimated loss to follow-up of $30 \%$ of patient participants, ${ }^{32}$ and the possibility that one cluster will drop out of each arm of the trial, 29 patient participants should be recruited in each centre, and one additional centre should be recruited and randomised to each arm. 
Therefore, for the definitive trial, initial estimates are that 348 patient participants should be recruited from 12 centres (6 centres in the intervention arm, and 6 centres in the control arm).

For the CHARMS pilot study, the number of patient participants recruited in each pilot centre will be slightly higher than the calculations for the definitive trial. One additional patient participant will be recruited in each pilot centre, bringing the total in each centre to 30. This slight increase is based on Viechtbauer et $a l \mathrm{~s}^{33}$ formula for the minimum sample size needed to detect unforeseen problems in a pilot study that will have a probability of occurring of 0.05 . As previously mentioned, patients' partners will also be invited to participate, in line with guidance on sexual assessment and counselling in cardiac rehabilitation. ${ }^{13}$ The research team will document the number of centres and the number of patients invited to achieve the required sample size, the numbers retained for analysis during the pilot and the time needed for recruitment.

\section{Recruitment}

Centre recruitment

All eligible centres listed in the directory of the Irish Association of Cardiac Rehabilitation ${ }^{26}$ will be invited to participate in the pilot. While the pilot is not a scale model of a cluster RCT, the invitation to participate in the pilot will mimic that of a full cluster RCT. In particular, it will specify that not all consenting centres will be offered the intervention. By inviting all eligible centres and by mimicking invitations to a full cluster RCT, we will obtain informative data on the feasibility of recruiting a sufficient number of centres to the definitive trial. We will document the number of centres that accept the invitation. Reasons for non-acceptance will also be documented.

We will stratify the consenting centres by location (in Dublin vs outside Dublin), and implement the pilot in one centre randomly selected from each stratum. This stratification strategy has been selected as Dublin accounts for one-quarter of the population of the Republic of Ireland, and Dublin differs from the rest of the country in terms of cultural, ethnic and religious diversity. ${ }^{34}$ All non-selected consenting centres will be invited to defer participation to the definitive trial (pending funding).

\section{Healthcare professional recruitment}

All health professionals at the selected centres will be invited to participate in the study. Study materials (invitation letters, information sheets, consent forms, questionnaires) will be given to the cardiac coordinator in each cardiac rehabilitation team by the CHARMS study researcher for distribution to his or her team, and will be returned directly to the CHARMS study researcher in two stamped addressed envelopes (the consent form and the questionnaire will be returned in separate envelopes to protect confidentiality). Staff members who do not wish to participate will be provided with the option of returning an opt-out card, which will allow for indication of reasons for non-participation. Data related to participation rates and reasons for non-participation will be recorded.

\section{Patient recruitment}

The cardiac coordinator at each participating centre will post study materials (invitation letters, information sheets, consent forms, questionnaires) to patients and partners starting phase III cardiac rehabilitation. Recruitment by post was deemed appropriate for two reasons. First, it would not put undue pressure on patients to participate in a potentially sensitive study. Second, as staff will for the most part be unaware if individual patients are participating in the study, it will help to minimise any conscious or unconscious differences in the treatment of patients based on participation.

Patients and partners will be asked to give consent to data collection only-as the CHARMS intervention is being delivered at centre level as a component of existing cardiac rehabilitation programmes. Consent forms and completed questionnaires will be returned directly to the CHARMS study researcher in stamped addressed envelopes (the consent form and the questionnaire will be returned in separate envelopes to protect confidentiality). Patients and partners who do not wish to participate will be provided with the option of returning an opt-out card, which will also allow for indication of reasons for non-participation. Data related to participation rates and reasons for non-participation will be recorded.

\section{Intervention procedures}

\section{The CHARMS staff intervention}

All staff at the participating centres will be invited to attend the CHARMS staff intervention, which will be delivered by a CHARMS Educator using a predeveloped manual, and will last for $\sim 2$ hours. The CHARMS staff intervention will include the following components: (1) welcomes, introductions and an initial outline of the nature of the CHARMS intervention; (2) rationale for the CHARMS intervention and introduction to international sexual counselling guidelines; ${ }^{13}$ (3) developing and practising of the sexual counselling skills; (4) an introduction to the CHARMS patient intervention; (5) creating an implementation plan for the CHARMS patient intervention, including nominating a staff member to deliver the patient intervention, and identifying and addressing barriers to implementation; (6) questions and answers. The CHARMS Educator will document the details of the implementation plan, and report the same to the research team. An example of the summary mapping of BCTs and intervention components is provided in the online supplementary file.

\section{The CHARMS patient intervention}

All patients will attend a structured intervention embedded into their cardiac rehabilitation programme (ie, as 
an additional group-based educational session) which will include (1) information on the impact of cardiovascular disease on sexuality; (2) information regarding a healthy sex life and communication strategies for use with a partner; (3) counselling on risks and fears regarding sex for people living with cardiovascular disease; (4) tips and strategies for resuming sex after a cardiac event, and for addressing sexual problems; (5) an invitation to request a one-to-one consultation and (6) time for questions and answers. The CHARMS patient intervention will be delivered by a member of the cardiac rehabilitation staff using a predeveloped manual, and will last for $30 \mathrm{~min}$. Partners of patients will also be invited to attend the intervention in line with international guidelines on the provision of sexual counselling, although it is acknowledged that some patients may prefer to attend it alone.

A patient information booklet will also be provided to patients. This booklet will contain information on the resumption of sexual activity after a cardiac event, and suggestions about how to deal with problems when they arise. Study posters will also be displayed in the participating clinics to reassure patients that a return to sexual activity after a cardiac event is normal, and encouraging patients to seek advice from staff if they have a sexual problem.

\section{Data collection}

When selected cardiac rehabilitation centres are recruited to the study, the research team will meet with cardiac rehabilitation staff to agree on how and when the CHARMS intervention will be delivered within their centre.

All quantitative study data will be gathered using printed questionnaires, which will be administered to participating health professionals, patients and partners. We will collect data at T1 (enrolment in cardiac rehabilitation/entry to the study), at T2 (3 months after entry to the study) and at T3 (6 months after entry to the study). Assessment at T2 will allow for detection of changes in measured outcomes postcompletion of the cardiac rehabilitation programme incorporating the CHARMS intervention. Assessment at T3 will allow for detection of persistence of these changes, and for detection of changes which may require a longer interval to occur.

\section{Staff questionnaires}

The measures below will be included in the staff questionnaire. All measures will be administered at T1, T2 and T3 (with the exception of demographic and professional background which will only be administered at T1).

Demographic and professional information: To include gender, age, profession, length of employment within cardiac rehabilitation, employment basis (full-time, parttime, other), whether the staff member is the cardiac coordinator, and details of employment in other cardiac rehabilitation centres.
Sexuality-related practice: To include the amount of time in the preceding 3 months devoted to sexual counselling activities, use of referrals to other services for the treatment of sexual problems, use of sexual counselling guidelines and perception of effectiveness with regard to sexual issues.

Barriers to discussing sexual problems: Adapted from Doherty $e t a l,{ }^{21}$ this measure includes 17 potential barriers to the provision of sexual counselling in cardiac rehabilitation (eg, I do not have enough time to discuss sexual problems). Responses are indicated on a Likert scale ranging from 1 (strongly disagree) to 5 (strongly agree).

Ratings of knowledge, awareness and confidence. Adapted from Doherty et $a l^{21}$ this measure includes three questions assessing knowledge, awareness and confidence in relation to the provision of sexual counselling (eg, How would you rate your awareness in dealing with sexual problems with cardiac rehabilitation patients?). Responses are indicated on a Likert scale ranging from 1 (poor) to 5 (excellent).

Sexual attitudes and beliefs: Adapted from Magnan $e t a l^{35}$ to be specific to cardiac rehabilitation, this measure includes 12 potential beliefs that staff may hold about the provision of sexual counselling (eg, Most cardiac rehab patients are too sick to be interested in sexuality). Responses are indicated on a Likert scale ranging from 1 (strongly disagree) to 6 (strongly agree).

The Sex after Myocardial Infarction Knowledge Test: The Sex after Myocardial Infarction Knowledge Test (SMIKT), developed by Steinke and Swan, ${ }^{36}$ includes 25 statements about the resumption of sex in the aftermath of a myocardial infarction (eg, Some medicines used for high blood pressure, anxiety or depression can affect sex). Possible responses to each statement are true, false and don't know.

Capability, opportunity and motivation: Informed by the $\mathrm{BCW},{ }^{22}$ this novel measure includes 10 items related to a staff member's self-rated capability, opportunity and motivation to provide sexual counselling to patients (eg, It is my responsibility to provide sexual counselling to my patients). Responses are indicated on a Likert scale ranging from 1 (strongly disagree) to 6 (strongly agree).

Feedback: Staff members will be invited to indicate the length of time it took to complete the questionnaire, and to provide any comments they have about participation in the study.

\section{Patient questionnaires}

The measures below will be included in the patient questionnaire. All measures will be administered at T1, T2 and T3. The same measures will be administered to partners, and at the same time points.

Demographic and medical information: To include gender, age, use of tobacco products, educational attainment, sexual orientation, cardiovascular diagnoses and procedures, relationship status, availability of health insurance, length of time since a cardiac event, use of sexual 
performance medications (eg, sildenafil) and use of cardiovascular medications.

The Sexual Self-Perception and Adjustment Questionnaire. The primary outcome for the study. Developed by Steinke et $a l^{30}$ this 28-item measure assesses sexual anxiety, sexual depression, sexual self-efficacy, sexual satisfaction and overall adjustment (eg, I am worried about having sexual activity). Responses are indicated on a Likert scale ranging from 1 (never) to 5 (always).

Health-related quality of life: Quality-adjusted life years associated with health state will be measured using the standardised EQ-5D-5L, ${ }^{37}$ which includes assessments of mobility, self-care, usual activities, pain/discomfort and anxiety/depression.

Cardiovascular-specific health-related quality of life. Developed specifically for patients with angina, myocardial infarction or ischaemic heart failure, this measure comprises 14 items assessing physical, emotional and global health-related quality of life (eg, In the last 4 weeks, have you been bothered by having to lift or move heavy objects?). Responses are indicated on a scale ranging from no to a lot.

Sexual activity and sexual problems: Adapted from Doherty $e t a l,{ }^{21}$ this measure includes assessments of the number of sexual partners, frequency of sex, desire for sex, presence of sexual problems and the use of professional services to address sexual problems.

Depression: Depressed mood and anhedonia will be measured using the 2-item Patient Health Questionnaire (PHQ-2), ${ }^{38}$ a widely used screening tool for depression.

Relationship satisfaction: Perceptions of relationship satisfaction will be assessed using the ENRICH scale. ${ }^{39}$ This is a 25-item scale (eg, My partner and I understand each other perfectly), with responses indicated on a Likert scale ranging from 1 (strongly disagree) to 5 (strongly agree).

Barriers to discussing sexual problems: Adapted from Doherty et $a l,{ }^{21}$ this measure includes 14 potential barriers to the discussion of sexual issues with healthcare providers during cardiac rehabilitation (eg, Cardiac rehab staff do not have enough time to discuss sexual problems). Responses are indicated on a Likert scale ranging from 1 (strongly disagree) to 6 (strongly agree).

The Sex after Myocardial Infarction Knowledge Test: As described above.

The International Index of Erectile Function: Male sexual function will be assessed with the 15-item International Index of Erectile Function (IIEF; eg, How do you rate your confidence that you could get and keep an erection?). ${ }^{40}$ The IIEF assesses five domains of male sexual function: erectile function, orgasmic function, sexual desire, intercourse satisfaction and overall satisfaction.

The Female Sexual Function Index: Female sexual function will be assessed with the 19-item Female Sexual Function Index (FSFI; eg, Over the past 4 weeks, how would you rate your level (degree) of discomfort or pain during or following vaginal penetration?). The FSFI assesses six domains of female sexual function: desire, arousal, lubrication, orgasm, satisfaction and pain.
Feedback: Patients and partners will be invited to indicate the length of time it took to complete the questionnaire, and to provide any comments they have about participation in the study.

\section{Pilot evaluation}

\section{Health economic analysis}

Without randomisation of centres to intervention and control arms of a trial, it is not possible to pilot a full economic evaluation whereby the costs and outcomes of alternative healthcare interventions are compared to determine cost-effectiveness. ${ }^{41}$ Instead, a cost-outcome description will be undertaken alongside the CHARMS pilot to examine the costs and outcomes of delivering the definitive intervention. ${ }^{41}$ The analysis will be undertaken in a manner consistent with the guidelines issued by the Health and Information Quality Authority in Ireland. ${ }^{42}$ Evidence will be collected on resource use and outcome measures at all time points of the pilot via patient questionnaires and other study data collection forms. With respect to costing, a publicly funded health service perspective will be adopted. Resource use associated with delivery of the intervention will be measured and costed, as will other resource use by patients over the course of the pilot study. In particular, the cost of intervention delivery will include costs relating to CHARMS Educator time input, production of training manuals, training room rental, rehabilitation staff time input and CHARMS patient consultations. Other resource use to be captured will include usage of medications, and attendance to primary care, community care and hospital care services over the course of the pilot study. Unit costs will be applied to convert data on resource use to resource costs, and a total cost variable will be calculated. The economic analysis will focus on the following two outcomes: (1) the primary outcome of the Sexual Self-Perception and Adjustment Questionnaire (SSPAQ) score; and (2) the economic outcome of quality-adjusted life years (QALY). The QALYs variable will be estimated using the EuroQol EQ-5D-5L survey instrument which will be completed by patients at all time points. ${ }^{37}$ The statistical analysis will explore the impact of the CHARMS intervention on the total cost and both outcome variables over the course of the pilot study. It will examine whether the CHARMS intervention is associated with an increase or decrease in total costs, and will further examine whether this is related to an increase or decrease in outcomes. Uncertainty in the analysis will be examined using a combination of standard statistical inference techniques, and univariate and multivariate sensitivity analyses.

\section{Evaluation of fidelity}

Fidelity of the CHARMS pilot study will be assessed using the guidance provided by the National Institute of Health's Behaviour Change Consortium. ${ }^{43}$ Fidelity assessment will provide valuable information on the feasibility of the intervention implementation, as well as 
information on the acceptability and usefulness of the fidelity assessment procedures which may require adaptation for the definitive intervention. ${ }^{23}$

Fidelity of the CHARMS staff intervention: A checklist will be developed for the distinct components of the staff intervention. The CHARMS Educator will review the checklist before delivering the staff intervention (as a reminder of the components to be delivered), and then complete the checklist after delivering the staff intervention (as a self-report of components actually delivered). Given that self-reported fidelity can be unreliable, ${ }^{44}$ an additional more objective method of assessment of the staff intervention will be audio recorded and the recording later independently coded by two members of the research team for the presence of the distinct components of the intervention, and also the associated BCTs used by the CHARMS Educator during delivery. ${ }^{45}$ The planned intervention components will be compared with those reported as delivered by the CHARMS Educator, and those coded as delivered by the researchers. ${ }^{46}$ Similarly, the planned BCTs will be compared with the BCTs coded as delivered by the researchers. The CHARMS Educator will also record the number of staff who attend the intervention and the number who did not attend, as well as the duration of the intervention. Fidelity of the staff intervention will be assessed in both cardiac rehabilitation centres participating in the CHARMS pilot by using the same standardised methods and materials in each centre.

Fidelity of the CHARMS patient intervention: A checklist will be developed for the distinct components of the patient intervention. The staff member delivering the patient intervention will review the checklist before delivering the intervention (as a reminder of the components to be delivered), and complete the checklist after delivering the intervention (as a self-report of components actually delivered). Patient attendance and duration of the intervention will be recorded by the staff member. The staff member will also distribute exit questionnaires to patients at the end of the intervention. Similar to the checklist provided to the staff member delivering the intervention, these exit questionnaires will ask patients to report on components delivered (eg, 'Did the staff member talk to your group about communication techniques?', allowing dichotomous yes/no responses), and also ask patients to report on how well they felt the components were delivered (eg, 'In your opinion, how well did the staff member explain the use of communication techniques', allowing responses on a Likert-type scale). In this manner, patient exit questionnaires will provide additional data on adherence (supplementing the self-report data provided by the staff member), and will also provide data on the competence of the staff member in delivering the intervention. ${ }^{45}$ As with the staff intervention, a more objective measure of fidelity will be provided by audio recording the patient intervention, and then having two researchers independently code the recording for the presence of the distinct components of the intervention. ${ }^{45}$ The planned intervention components will be compared with the components reported as delivered by the CHARMS Educator, those reported as delivered by the patients, and those coded as delivered by the researchers. ${ }^{46}$ Similarly, the planned BCTs will be compared with the BCTs coded as delivered by the researchers. Fidelity of the patient intervention will be assessed in both participating cardiac rehabilitation centres by using the same standardised methods and materials in each centre.

To maximise fidelity, the staff member will be provided with an intervention pack that includes all materials necessary to effectively deliver the patient intervention. This pack will include predelivery and postdelivery checklists, a staff manual, patient booklets and exit questionnaires for patients. To promote the maintenance of skills and adherence to the intervention over time, the research team will provide a number of supports. A schedule of phone calls (eg, weekly or bimonthly) between the research team and a designated member of staff will be agreed upon, so that any issues related to the intervention delivery can be reported, documented and addressed. Staff members can request additional intervention packs (by phone or online), and resources such as manuals and training videos will be available for download on the study website. The research team will also document the details of how the CHARMS patient intervention is incorporated into the cardiac rehabilitation programme at each participating centre, the number of contacts between staff and the research team during the intervention, and the numbers of sex-related queries, discussions and consultations recorded by staff with patients. Fidelity of the intervention will also be further explored in qualitative interviews conducted with staff and patients, as described in more detail below.

\section{Quantitative evaluation of feasibility and acceptability}

We will document the number of invitations sent to cardiac rehabilitation centres, the number of refusals and the number of acceptances. We will document the same for staff, patients and partners. Attrition (if any) of centres, staff or patients/partners will be documented at each data collection time point. Levels of missing data in returned questionnaires will be reported. Questionnaires administered to staff, patients and partners at all data collection time points will include a comments section, where participants will be invited to comment on the comprehensibility and acceptability of the questionnaires, how the questionnaires might be improved, and how long these took to complete.

\section{Qualitative evaluation of feasibility, acceptability and fidelity}

Using a descriptive qualitative methodology, all cardiac rehabilitation staff will be invited to participate in semistructured interviews assessing their perspectives and experiences of the CHARMS intervention. The interview guide will include questions related to the experience of 
participating in the study, the feasibility and acceptability of the study procedures, and the perceived effectiveness of the intervention. Additionally, the interview guide will further explore the fidelity of the intervention, including the nature of and rationale for any adaptations made to the intervention, beliefs about the quality of intervention delivery, the need for supports to maintain sexual counselling skills over time and the identification of additional training needs. Staff members will be interviewed at a single time point in the second half of the data collection period. This will allow for sufficient time to ensure that staff have had experience of delivering the patient intervention, and also experience handling requests for one-to-one support from patients.

A qualitative longitudinal approach will be used to capture patients' perspectives and experiences of the CHARMS intervention, and how and why these perspectives and experiences change over time. ${ }^{47}$ Purposive sampling will be employed by selecting patients for interview based on data from the study measures, and use of data from exit interviews administered at the end of the patient intervention (eg, purposively sampling patients with high and low SSPAQ scores, patients reporting and not reporting organic sexual dysfunction, patients with positive and negative evaluations in exit interviews, etc). This will allow for maximum variation in the sample in terms of demographic characteristics, study outcomes and experiences of the intervention. Semistructured interviews will be conducted with 30 patients in total, 15 from each participating centre. The 30 patients will be interviewed at T2, and again at T3. Interviews at T2 will explore the patients' initial experiences of participating in the study and initial usage of the intervention content. Interviews at T3 will focus on ascertaining patients' use of the intervention content over time.

Qualitative analysis: Staff and patient interviews will be transcribed verbatim and entered into the qualitative analysis software program NVivo to facilitate data management, coding and retrieval. (NVivo qualitative data analysis software [program]. 10 version, 2012). All data will be initially open coded using thematic analysis, and then axial coded to identify categories. ${ }^{48}$ Longitudinal data from individual patients will be analysed within time (ie, at T2 and then at T3), and also across time (ie, comparing T2 to T3) to capture changes for these individuals. A thematic analysis of these changes will then be produced, incorporating data from all patients. This longitudinal analysis will be guided by Holland et al. ${ }^{49}$ In addition, the framework for the interrogation of longitudinal qualitative data as proposed by Lewis, ${ }^{50}$ and Saldaña's ${ }^{51}$ guide to the qualitative analysis of change through time will inform the analysis. The rigour of the analysis will be maintained using the criteria of credibility, audibility, confirmability and applicability. ${ }^{52}$

\section{Statistical analysis}

A study statistical database (using SPSS Data Collection V.7 software) will be created by the study manager and overseen by the study statistician; questionnaire responses will be entered. Suitable graphical and numerical summaries will be generated for the staff and patient measures at baseline. The aim of this pilot study is to inform the development of a definitive trial; although the small sample size will preclude effectiveness being established, the primary outcome measure (SSPAQ) will be analysed to mimic the definitive trial. Linear mixed models will be used to model the longitudinal change for the primary and secondary outcomes, while adjusting for cluster membership and within-participant correlation over time. Tree-based models for longitudinal responses will be used to uncover potentially useful relationships in data that may be informative when designing the definitive trial. All analyses of the primary outcome will be treated as preliminary and interpreted with caution. An estimate of the likely effect size will be calculated, as well as an estimate of the intra-cluster correlation.

\section{ETHICS AND DISSEMINATION}

This study will be conducted in accordance with the ethical principles derived from the Declaration of Helsinki. ${ }^{53}$ Written informed consent will be obtained from all participants. Study personnel will be covered by indemnity for negligent harm through standard arrangements in the National University of Ireland (NUI) Galway, and through standard arrangements at the selected cardiac rehabilitation centres. In the event of amendments to the protocol, relevant Research Ethics Committees (RECs) will be notified, approval for amendments obtained and appropriate study materials updated. Findings will be disseminated in peer-reviewed journals and presented at conferences to publicise the outcomes to cardiac rehabilitation staff, patients and relevant policymakers.

\section{Author affiliations}

${ }^{1}$ Health Behaviour Change Research Group, School of Psychology, NUI Galway, Ireland

${ }^{2}$ School of Nursing and Midwifery, NUI Galway, Ireland

${ }^{3}$ Department of Psychology, Royal College of Surgeons in Ireland, Dublin, Ireland

${ }^{4}$ School of Business and Economics, NUI Galway, Ireland

${ }^{5}$ Department of Social and Welfare Studies, Linköping University, Norrköping,

Sweden

${ }^{6}$ Department of General Practice, NUI Galway, Ireland

${ }^{7}$ HRB Clinical Research Facility, NUI Galway, Ireland

${ }^{8}$ School of Nursing, Wichita State University, Kansas, USA

${ }^{9}$ School of Public Health, Physiotherapy and Sports Science, University

College Dublin, Ireland

Acknowledgements This work was supported by the Health Research Board grant number HRA-2014-HSR-519.

Contributors MB is the principal investigator, and led the pilot study design and funding application. PJM is the pilot study project manager; he contributed to study design and wrote the first draft of the manuscript. DC, $\mathrm{SD}, \mathrm{TJ}, \mathrm{AWM}, \mathrm{JN}, \mathrm{MO}$ and EES have all contributed to the study design, and are co-applicants on the funding application. JM offered particular input in relation to intervention development. DC offered particular input in relation to the qualitative research. PG is a health economist and contributed to the health economic analysis. JN is the study statistician and contributed to the 
statistical aspects of the protocol. ET offered particular input in relation to the intervention fidelity assessment strategy. All authors contributed to, read and approved the final version of the manuscript.

Funding Health Research Board.

Competing interests None declared.

Ethics approval Research Ethics Committee (REC) of the National University of Ireland, Galway. Approval will also be sought from the relevant RECs in participating hospitals.

Provenance and peer review Not commissioned; externally peer reviewed.

Open Access This is an Open Access article distributed in accordance with the Creative Commons Attribution Non Commercial (CC BY-NC 4.0) license, which permits others to distribute, remix, adapt, build upon this work noncommercially, and license their derivative works on different terms, provided the original work is properly cited and the use is non-commercial. See: http:// creativecommons.org/licenses/by-nc/4.0/

\section{REFERENCES}

1. World Health Organisation. Cardiovascular Diseases (CVDs). 2015 http://www.who.int/mediacentre/factsheets/fs317/en/

2. Schumann J, Zellweger MJ, Di Valentino M, et al. Sexual dysfunction before and after cardiac rehabilitation. Rehabil Res Pract 2010;2010:823060.

3. Kütmeç C, Yurtsever S. Effects of sexual function of essential hypertensions in Women. Eur J Cardiovasc Nurs 2011:10:56-63.

4. Træen B, Olsen S. Sexual dysfunction and sexual well-being in people with heart disease. Sex Relationship Ther 2007;22:193-208.

5. Günzler C, Kriston L, Harms A, et al. Association of sexual functioning and quality of partnership in patients in cardiovascular rehabilitation-a gender perspective. J Sex Med 2009;6:164-74.

6. O'Farrell P, Murray J, Hotz SB. Psychologic distress among spouses of patients undergoing cardiac rehabilitation. Heart Lung 2000;29:97-104.

7. Jackson G, Rosen RC, Kloner RA, et al. The second Princeton consensus on sexual dysfunction and cardiac risk: new guidelines for sexual medicine. J Sex Med 2006;3:28-36; discussion 36.

8. Mandras SA, Uber PA, Mehra MR. Sexual activity and chronic heart failure. Mayo Clinic Proceedings Vol. 82. No. 10. Elsevier, 2007.

9. Mosack V, Steinke EE. Trends in sexual concerns after myocardial infarction. J Cardiovasc Nurs 2009;24:162-70.

10. Sasayama S, Ishii N, Ishikura F, et al. Men's Health Study: epidemiology of erectile dysfunction and cardiovascular disease. Circ J 2003;67:656-9.

11. Nicolai MPJ, Liem SS, Both S, et al. A review of the positive and negative effects of cardiovascular drugs on sexual function: a proposed table for use in clinical practice. Neth Heart $J$ 2014;22:11-19.

12. Levine GN, Steinke EE, Bakaeen FG, et al., American Heart Association Council on Clinical Cardiology; Council on Cardiovascular Nursing; Council on Cardiovascular Surgery and Anesthesia; Council on Quality of Care and Outcomes Research. Sexual activity and cardiovascular disease: a scientific statement from the American Heart Association. Circulation 2012;125:1058-72.

13. Steinke EE, Jaarsma T, Barnason SA, et al., Council on Cardiovascular and Stroke Nursing of the American Heart Association and the ESC Council on Cardiovascular Nursing and Allied Professions (CCNAP). Sexual counselling for individuals with cardiovascular disease and their partners: a consensus document from the American Heart Association and the ESC Council on Cardiovascular Nursing and Allied Professions (CCNAP). Circulation 2013;128:2075-96.

14. D'Eath M, Byrne M, Doherty S, et al. The Cardiac Health and Assessment of Relationship Management and Sexuality study: a qualitative inquiry of patient, general practitioner, and cardiac rehabilitation staff views on sexual assessment and counseling for cardiac patients. J Cardiovasc Nurs 2013;28:E1-13.

15. Jaarsma T, Steinke EE, Gianotten WL. Sexual problems in cardiac patients: how to assess, when to refer. J Cardiovasc Nurs 2010;25:159-64.

16. Klein R, Bar-on E, Klein J, et al. The impact of sexual therapy on patients after cardiac events participating in a cardiac rehabilitation program. Eur J Cardiovasc Prev Rehabil 2007;14:672-8.

17. Levine GN, Steinke EE, Bakaeen FG, et al. Sexual activity and cardiovascular disease a scientific statement from the American Heart Association. Circulation 2012;125:1058-72.
18. Byrne M, Doherty S, Murphy AW, et al. The CHARMS Study: cardiac patients' experiences of sexual problems following cardiac rehabilitation. Eur J Cardiovasc Nurs 2013;12:558-66.

19. Layte RD, McGee HP, Quail A, et al. The Irish study of sexual health and relationships health among young people In Ireland main report. Crisis Pregnancy Agency, Department of Health and Children (DOHC), 2006.

20. Byrne M, Doherty S, McGee HM, et al. General practitioner views about discussing sexual issues with patients with coronary heart disease: a national survey in Ireland. BMC Fam Pract 2010;11:40.

21. Doherty S, Byrne M, Murphy AW, et al. Cardiac rehabilitation staff views about discussing sexual issues with coronary heart disease patients: a national survey in Ireland. Eur J Cardiovasc Nurs 2011;10:101-7.

22. Michie S, Atkins L, West R. The behaviour change wheel: a guide to designing interventions. Great Britain: Silverback Publishing, 2014

23. Craig $\mathrm{P}$, Dieppe $\mathrm{P}$, Macintyre $\mathrm{S}$, et al. Developing and evaluating complex interventions: the new Medical Research Council guidance. Int J Nurs Stud 2013;50:587-92.

24. Richards DA, Hallberg IR. Complex interventions in health: an overview of research methods. Routledge, 2015.

25. Arain M, Campbell MJ, Cooper CL, et al. What is a pilot or feasibility study? A review of current practice and editorial policy. BMC Med Res Methodol 2010;10:67.

26. Irish Association of Cardiac Rehabilitation. Directory 2014. http:// www.iacr.info/wp-content/uploads/2015/03/2014Directory3.pdf

27. Lindau ST, Abramsohn E, Gosch K, et al. Patterns and loss of sexual activity in the year following hospitalization for acute myocardial infarction (a United States National Multisite Observational Study). Am J Cardiol 2012;109:1439-44.

28. Lindau ST, Schumm LP, Laumann EO, et al. A study of sexuality and health among older adults in the United States. N Engl J Med 2007:357:762-74

29. Coleman E. Masturbation as a means of achieving sexual health J Psychol Hum Sex 2003;14:5-16.

30. Steinke EE, Mosack V, Hill TJ. Sexual self-perception and adjustment of cardiac patients: a psychometric analysis. $J$ Res Nurs 2013;18:191-201.

31. Hemming K, Girling AJ, Sitch AJ, et al. Sample size calculations for cluster randomised controlled trials with a fixed number of clusters. BMC Med Res Methodol 2011;11:102.

32. Cupples ME, McKnight A. Five year follow up of patients at high cardiovascular risk who took part in randomised controlled trial of health promotion. BMJ 1999;319:687-8

33. Viechtbauer W, Smits L, Kotz D, et al. A simple formula for the calculation of sample size in pilot studies. $J$ Clin Epidemiol 2015:68:1375-9.

34. Central Statistics Office. This is Ireland: highlights from Census 2011, part 1. Dublin, Ireland: Central Statistics Office, 2012. http:// www.cso.ie/en/media/csoie/census/documents/census2011pdr/ Census,2011,Highlights,Part, 1,web,72dpi.pdf

35. Magnan MA, Reynolds KE, Galvin EA. Barriers to addressing patient sexuality in nursing practice. Medsurg Nurs 2005;14: 282-90.

36. Steinke EE, Swan JH. Effectiveness of a videotape for sexual counseling after myocardial infarction. Res Nurs Health 2004:27:269-80.

37. EuroQol Group. EuroQol-a new facility for the measurement of health-related quality of life. Health Policy 1990;16:199-208.

38. Kroenke K, Spitzer RL, Williams JB. The Patient Health Questionnaire-2: validity of a two-item depression screener. Med Care 2003;41:1284-92.

39. Fowers BJ, Olson DH. ENRICH marital satisfaction scale: a brief research and clinical tool. J Fam Psychol 1993;7:176.

40. Rosen RC, Riley A, Wagner G, et al. The international index of erectile function (IIEF): a multidimensional scale for assessment of erectile dysfunction. Urology 1997;49:822-30.

41. Drummond M, Sculpher M, Claxton K, et al. Methods for the economic evaluation of health care programmes. Oxford: Oxford University Press, 2015

42. Health Information and Quality Authority. Guidelines for the Economic Evaluation of Health Technologies in Ireland 2014. http://www.hiqa.ie/publication/guidelines-economic-evaluation-healthtechnologies-ireland

43. Borrelli B. The assessment, monitoring, and enhancement of treatment fidelity in public health clinical trials. J Public Health Dent 2011;71:S52-63.

44. Hardeman W, Michie S, Fanshawe T, et al. Fidelity of delivery of a physical activity intervention: predictors and consequences. Psychol Health 2008;23:11-24. 
45. Breitenstein SM, Gross D, Garvey CA, et al. Implementation fidelity in community-based interventions. Res Nurs Health 2010;33:164-73.

46. French SD, Green SE, Francis JJ, et al. Evaluation of the fidelity of an interactive face-to-face educational intervention to improve general practitioner management of back pain. BMJ Open 2015;5: e007886.

47. Corden A, Millar J. Qualitative longitudinal research for social policyintroduction to themed section. Soc Policy Soc 2007;6:529-32.

48. Corbin J, Strauss A. Basics of qualitative research: techniques and procedures for developing grounded theory. Sage publications, 2014.
49. Holland J, Thomson R, Henderson S. Qualitative longitudinal research: a discussion paper. London South Bank University London, 2006.

50. Lewis J. Analysing qualitative longitudinal research in evaluations. Soc Policy Soc 2007;6:545-56.

51. Saldaña J. Longitudinal qualitative research: analyzing change through time. Rowman Altamira, 2003.

52. Lincoln YS, Guba EG. Naturalistic inquiry. Sage, 1985.

53. World Medical Association. World Medical Association Declaration of Helsinki: ethical principles for medical research involving human subjects. JAMA 2013;310:2191-4. 